\title{
Espirulina un suplemento alimenticio como posible alternativa en el control de peso. Un estudio con ratas Wistar
}

\section{Spirulina a nutritional supplement as a possible alternative in weight control.}

\section{A study with Wistar rats}

\author{
Guillen-Martín del Campo Jorge Alberto, Calvillo-Femat Antonio, Mosqueda-Esparza Joyce Ivonne, \\ Rodríguez-Hernández Adán Israel*, Jaramillo-González Francisco
}

\begin{tabular}{l} 
Datos del Artículo \\
\hline Universidad Autónoma de Aguascalientes. \\
Departamento de Nutrición y Cultura Física. \\
Av. Universidad \# 940. \\
Ciudad Universitaria. \\
C.P. 20131 , Aguascalientes. \\
México. \\
Tel: +52 4499107400. Ext 410. \\
*Dirección de contacto: \\
Universidad Autónoma de Aguascalientes. \\
Departamento de Nutrición. \\
Av. Universidad \# 940. \\
Ciudad Universitaria. \\
C. P. 20131, Aguascalientes. \\
México. \\
Tel: (066)312510-(066)312230 \\
Móvil: 966881161 \\
Adán Israel Rodríguez-Hernández \\
E-mail address: israel.rodriguez@edu.uaa.mx \\
adan_israelll@yahoo.com.mx
\end{tabular}

\section{Palabras clave:}

Alga espirulina (Arthrospira sp.),

J. Selva Andina Res. Soc. 2020; 11(1):49-56.

\section{Historial del artículo.}

Recibido agosto 2019

Devuelto octubre 2019

Aceptado noviembre 2019

Disponible en línea, febrero 2020.

\section{Editado por:}

Selva Andina

Research Society

\section{Key words:}

Spirulina Alga,

obesity,

weight control.

Wistar rats

\section{Resumen}

Recientemente el alga espirulina ha sido utilizada para reducción de peso. Se sabe que al menos $36 \mathrm{~g}$ de esta alga aportan todos aminoácidos esenciales, entre otros diferentes compuestos como fibra y flavonoides con la posibilidad de tratar la obesidad o el sobrepeso, El objetivo fue determinar si la administración de espirulina ayuda a reducir el peso corporal en ratas Wistar así como determinar posibles efectos en el metabolismo de diferentes marcadores generales bioquímicos como lo fue un perfil lipídico, urea y creatinina. Se aplicó un estudio experimental doble ciego empleando 10 ratas Wistar, cuatro ratas denominadas experimentales, se les administró 5.8 mg de espirulina diluida en $1 \mathrm{~mL}$ de agua, por sonda orogástrica durante ocho semanas, al grupo control (6 ratas misma especie y peso inicial) se le administró únicamente $1 \mathrm{~mL}$ de agua. El peso de las ratas experimentales versus las control no tuvo cambios significativos. Respecto a los marcadores bioquímicos únicamente se encontró un aumento significativo de los valores de urea, colesterol total, y triglicéridos del grupo experimental en relación al control. Los hallazgos sugieren a la ingesta de espirulina para el control de peso y en posibles estados de malnutrición.

(C) 2020. Journal of the Selva Andina Research Society. Bolivia. Todos los derechos reservados.

\section{Abstract}

Recently spirulina algae have been used for weight reduction. It is known that at least $36 \mathrm{~g}$ of this algae provide all the essential amino acids, among other different compounds such as fiber and flavonoids with the possibility of treating obesity or overweight, The objective was to determine if the administration of spirulina helps reduce body weight in Wistar rats as well as determining possible effects on the metabolism of different general biochemical markers such as a lipid profile, urea and creatinine. A double-blind experimental study was applied using 10 Wistar rats, four rats called experimental rats, $5.8 \mathrm{mg}$ of spirulina diluted in $1 \mathrm{~mL}$ of water, by orogastric tube for eight weeks, were administered to the control group ( 6 rats same species and initial weight) He was given only $1 \mathrm{~mL}$ of water. The weight of the experimental rats versus the controls had no significant changes. Regarding the biochemical markers, only a significant increase in the values of urea, total cholesterol, and triglycerides of the experimental group in relation to the control was found. The findings suggest the intake of spirulina for weight control and in possible states of malnutrition. 


\section{Introducción}

Desde el punto de vista del tema que nos ocupa, es indudable que han predominado aspectos negativos en nuestros hábitos, es decir, no hemos sido capaces de cambiar la forma de comer, movernos y, como resultado, tenemos ante nosotros la «obesidad», una enfermedad que representa un gran problema de salud que afecta a países desarrollados y en vías de desarrollo ${ }^{1}$.

El alga Spirulina sp. (Arthrospira sp.), una cianobacteria filamentosa no diferenciada, habitante de lagos alcalinos, cultivada para consumo humano por su contenido nutricional ${ }^{2}$. Desde el punto de vista nutricional, las algas se caracterizan por presentar bajo contenido calórico, ya que, aunque presentan elevado porcentaje de proteínas, la cantidad de lípidos es reducida, una gran parte de carbohidratos, polisacáridos no digeribles por enzimas digestivas humanas ${ }^{3}$.

El valor de la espirulina (ES) radica precisamente en la gran variedad de macro y micronutrientes contenidos, así como algunas de sus propiedades como el incrementar niveles energéticos, por las vitaminas que participan en el metabolismo, como cofactores, además de reducir el estrés premenstrual, incrementar el rendimiento en atletas, ofrecer protección antioxidante ${ }^{4}$.

Esta cianobacteria como fuente rica de proteínas, aminoácidos, vitaminas, minerales y otros nutrientes de ahí sus principales usos como suplemento alimenticio, sea en polvo, encapsulado, tabletas, barras de granola, golosinas o bebidas instantáneas de frutas o vegetales ${ }^{4}$. Los metabolitos de interés biotecnológico son entonces: los carotenoides y $\beta$ carotenos, ficobiliproteinas, exopolisacáridos, lípidos, proteínas vitaminas, minerales que contiene ${ }^{4}$.
La ES contiene alrededor del $65 \%$ de proteínas, proporción superior al de otras fuentes proteicas, carece de celulosa dura, proporcionando una mejor digestibilidad, factor importante en individuos con malabsorción intestinal. A demás, de ser rica en vitaminas $\mathrm{A}, \mathrm{E}, \mathrm{B}_{12}$, ácido gama linolénico y hierro $^{4}$.

Con el tiempo la espirulina ha sido estudiada como útil para la prevención de enfermedades cardiovasculares y control de peso ${ }^{4}$. Sin embargo, tiempo atrás esta alga comenzó a ser recomendada para la pérdida de peso (PDP) con el argumento de presentar propiedades anorexigénicas, desde los años 80 's ${ }^{5}$.

Por otro lado, Hirahashi et $\mathrm{al}^{6}$ reporto que su ingesta involucra el incremento de células natural killler, fortaleciendo de esta manera el sistema inmune, así mismo se ha descrito su potencial antioxidante en la prevención de enfermedades neurodegeneraticas ${ }^{7}$.

Aunque la base terapéutica fundamental para obtener un peso adecuado es la dieta hipocalórica personalizada, con el incremento de la actividad física, la utilización de fármacos tiene sus indicaciones precisas durante el tratamiento de la PDP $^{8}$. Éstos han sido fuertemente cuestionados, junto con otros productos comercializados, y su posible relación con la obesidad. Sin embargo, en los últimos años nuevos alimentos pretenden ir más allá ${ }^{8,9}$.

Por otro lado, esta alga ha sido propuesta para estados de desnutrición, por la amplia gama de nutrientes, vitaminas $y$ minerales ${ }^{10}$. Se ha comprobado a nivel experimental in vivo e in vitro su efectividad en el tratamiento de algunos tipos de alergias, anemia, cáncer, hepatotóxicidad, enfermedades virales, cardiovasculares, hiperglicemia, hiperlipémia, inmunodeficiencia y procesos inflamatorios, entre otros ${ }^{11}$. 
Las propiedades comprobadas: Antialérgico. Protege contra el desarrollo de alergias, disminuyendo las reacciones anafilácticas, por el bloqueo en la liberación de histamina y del factor de necrosis tumoral (TNF-a) inducida por $\operatorname{IgE}^{11}$. Antihepatotóxico. Disminuye la concentración de triacilgliceroles séricos, un efecto hepatotóxico, relacionado con la producción de radicales libres, es posible que su potencial hepatoprotector de ES máxima, se deba a sus constituyentes tales como ácido gamma-linolénico, sulfolípidos, selenio, clorofila, caroteno y vitaminas $\mathrm{E}$ y C. El ácido gamma-linolénico y los sulfolípidos han sido también implicados en otras propiedades farmacológicas ${ }^{11}$. Efectos prebióticos. Se reportó en diversas pruebas que a cierta dosis de ES puede inducir el crecimiento microbiano, y sus productos metabólicos, de especies, Lactobacillus y Lactococus lactis ${ }^{12}$. Antiviral. Diversos autores han señalado que los diferentes compuesto bioactivos, entre ellos la ficocianina, son responsables de inhibir ciertos virus $2,12,13,14$. Antioxidante. Contiene ingredientes activos como el phycocyanin y el $\beta$-caroteno que poseen una potente actividad antioxidante y antiinflamatoria. El phycocyanin tiene la capacidad de atrapar radicales libres ${ }^{3,15-17}$.

Sus propiedades y aplicaciones hacen de este alimento "promotor de la salud" o "nutracéutico"4. Incluso se recomienda en practicantes del vegetarianismo ${ }^{14,15}$, además que ha señalado ser un buen sustituto de la vitamina $\mathrm{B}_{12}$.

Otros tipos de alga verde azul podrían algunas veces secretar sustancias hepatotóxicas y/o neurotóxicas, o albergar bacterias como el Vibrio cholerae ${ }^{4}$. Por el contrario, en esta especie ha descrito no tener efectos tóxicos o teratógenos en animales de estudio ${ }^{18}$. La ES también podría concentrar metales pesados como plomo y mercurio, o patógenos potenciales ${ }^{4,7}$
La ES que se comercializa, contiene proteínas cuyo principal aminoácido, la fenilalanina, además trazas de minerales como el zinc y magnesio, vitaminas del complejo B, betacarotenos, ácido fólico y calcio $^{4,19-21}$. Se ha usado incluso por astronautas para prevenir deficiencias nutricionales ${ }^{2}$, inclusive con fines terapéuticos antiinflamatorios ${ }^{16,22}$.

Nuevas contribuciones de estudios respecto al control de peso con ES revelan efectos positivos en la reducción de peso $(\mathrm{RP})^{23}$, mientras que a su vez, se han estudiado los mecanismos moleculares y enzimáticos de los genes SIRT1 y AMPk implicados tras la ingesta de ES favoreciendo específicamente también la $\mathrm{RP}^{24}$. Un estudio de revisión reciente basado en estudios in vitro e in vivo en animales menciona la importancia de su uso a nivel farmacéutico con efecto antiinflamatorio, anti-obesidad, anti-diabético entre otros ${ }^{21}$. Sotiroudis \& Sotiroudis $^{25}$ hace mención a que esta alga ha sido aceptada para el consumo humano sin riesgos a la salud según el Comité de Expertos en Información de Suplementos Dietéticos de la Convención Farmacopeica de los Estados Unidos, sin embargo, algunos casos han reportado efectos adversos ${ }^{21}$. Mientras en el campo de la salud se ha visto resultados amplios por su riqueza nutrimental, tanto en compuestos bioactivos como ficocianina, tocoferol, $\beta$-caroteno, ácidos caféicos y clorogénico han sido propuestos para el tratamiento complementario en enfermedades como diabetes, dislipidemia, ulcera, infecciones recurrentes ${ }^{22,26}$, incluso en la ganancia de masa muscular en atletas ${ }^{16}$.

El objetivo de este estudio fue determinar si existe asociación del consumo de esta alga con la reducción de peso y determinar posibles efectos en el metabolismo de diferentes marcadores generales bioquímicos como lo es un perfil lipídico, urea y creatinina. 


\section{Materiales y métodos}

Modelo experimental in vivo. Se trabajó con ratas de la línea parda de la especie Wistar de la Universidad Autónoma de Aguascalientes (UAAC), que se instalaron en su bioterio, fueron aclimatadas durante 8 semanas a temperatura de $25 \pm 2{ }^{\circ} \mathrm{C}$ con una humedad relativa de $65 \pm 5 \%$ y un ciclo de oscuridad $12 / 12 \mathrm{~h}$, se las mantuvo con libre acceso a agua y alimento balanceado (ad libitum) ${ }^{27}$. Tras un muestreo aleatorio simple a 12 ratas Wistar hembras adultas, 6 del grupo experimental (GE) fueron tratadas con la administración de una solución de alga ES marca Pronat Ultra por la vía orogástrica durante 8 semanas, sin embargo, durante la investigación fallecieron 2 ratas del GE por intoxicación con éter, obteniéndose una población final 10 ratas, 4 de ellas ratas experimentales, al grupo control solo le administró $1 \mathrm{~mL}$ de agua. Todas las ratas tenían un peso promedio inicial por grupo de $180 \mathrm{~g}$ cada una, y 6 ratas controles con un peso promedio inicial por grupo de $193 \mathrm{~g}$ (los pesos iniciales por cada rata se muestran en la tabla 1). El pesaje se realizó diariamente, al final del estudio las ratas fueron exsanguinadas. Los valores se compararon con el rango de valores de normalidad, según la base de datos para ratas albinas macho (glucosa $90-130 \mathrm{mg} / \mathrm{dL}$, colesterol 160-220 mg/dL, triglicéridos 123-186 mg/dL) ${ }^{28}$. Equipamiento. La balanza utilizada fue una granataria de 3 pilotes con pesas modelo BAL-3 la marca Zeigen. Para determinar los niveles de glucosa se utilizó el kit analizador miltiparámetro 3 en 1 Multicare-in (Laboratorio Biochemical Systems International), y se usó un espectrofotómetro de la marca Clima Plus.

Como procedimiento de eutanasia, respecto a las recomendaciones para el manejo de animales de experimentación según la Directiva Europea 2010/63/EU con la técnica de dislocación cervical. Análisis estadístico. Se utilizó un análisis estadístico mediante la prueba t de Student con una $\mathrm{P}=<0.05$ (la cual se compara con una $t$ con 8 grados de libertad equivalente a 1.860), se consideró con significancia estadística para la medición de cambio de las variables peso, colesterol total, colesterol LDL, colesterol HDL, triglicéridos, urea y creatinina en suero.

\section{Resultados}

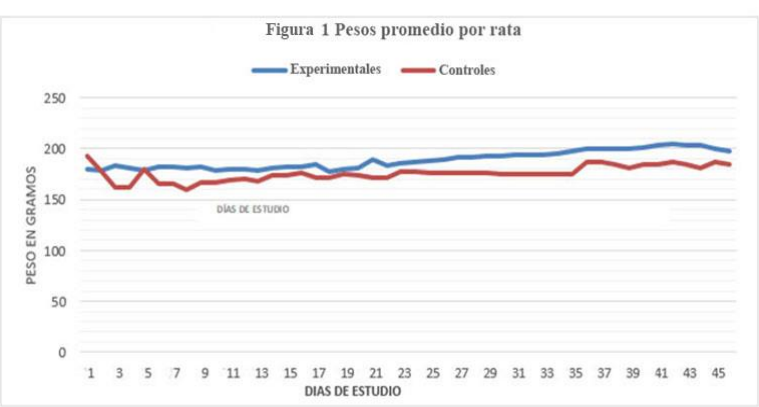

En la figura 1, se observa el cambio de peso de las ratas a partir del día 5, el peso promedio del grupo control fue de $165 \mathrm{~g}$ y experimental $178 \mathrm{~g}$. El peso mayor del GE se mantuvo entre los días 41 a 44 y el mayor para el grupo control (GC) es el día 1.

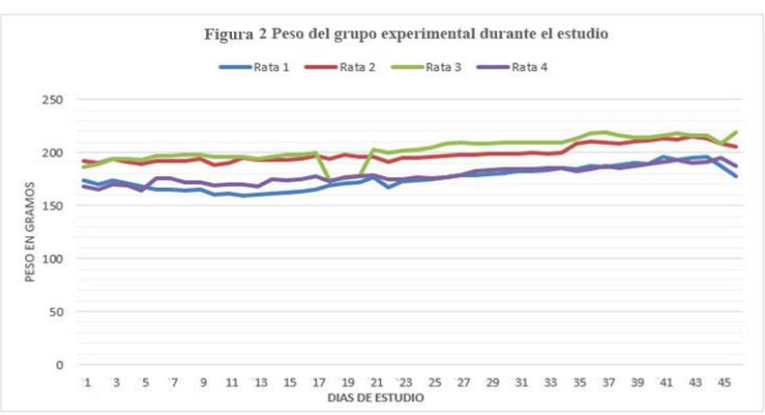

La tabla 1 se presenta las diferencias del peso de las ratas experimentales y controles al inicio y al final del estudio.

En la tabla 2 se observan los pesos promedio resultantes de los dos grupos durante el tiempo total del estudio. 
Tabla 1 Pesos iniciales y finales por rata experimentales y controles

\begin{tabular}{cccccc}
\hline $\begin{array}{c}\text { Grupo } \\
\text { experimental }\end{array}$ & Peso inicial (g) & Peso final (g) & $\begin{array}{c}\text { Grupo } \\
\text { control }\end{array}$ & Peso inicial (g) & Peso final (g) \\
\hline Rata 1 & 174 & 178 & Rata 1 & 199 & 172 \\
Rata 2 & 192 & 206 & Rata 2 & 188 & 190 \\
Rata 3 & 186 & 219 & Rata 3 & 201 & 205 \\
Rata 4 & 168 & 187 & Rata 4 & 195 & 185 \\
& & & Rata 5 & 187 & 178 \\
& & & Rata 6 & 190 & 193 \\
\hline
\end{tabular}

Tabla 2 Pesos promedio durante el estudio por ratas experimentales y controles

\begin{tabular}{cccc}
\hline $\begin{array}{c}\text { Grupo } \\
\text { experimental }\end{array}$ & Peso promedio $(\mathbf{g})$ & $\begin{array}{c}\text { Grupo } \\
\text { control }\end{array}$ & $\begin{array}{c}\text { Peso } \\
\text { promedio (g) }\end{array}$ \\
\hline Rata 1 & 175.69 & Rata 1 & 169.78 \\
Rata 2 & 198.75 & Rata 2 & 178.60 \\
Rata 3 & 202.80 & Rata 3 & 186.16 \\
Rata 4 & 178.86 & Rata 4 & 174.52 \\
& & Rata 5 & 166.86 \\
& & Rata 6 & 178.50 \\
\hline
\end{tabular}

Tabla 3 Comparación de química sanguínea al final del estudio por grupo experimentales y controles

\begin{tabular}{llcccc}
\hline \multicolumn{1}{c}{ Variable } & \multicolumn{1}{c}{ Media } & & SD & T & P \\
\hline \multirow{2}{*}{ Peso } & Experimentales & 189.0 & 13.7 & \multirow{2}{*}{0.79} & 0.148 \\
& Controles & 175.74 & 6.93 & & \\
Urea & Experimentales & 38.60 & 4.51 & 3.04 & 0.029 \\
& Controles & 32.00 & 2.80 & & \\
Creatinina & Experimentales & 0.540 & 0.195 & -1.03 & 0.327 \\
& Controles & 7.8 & 24.3 & & \\
Colesterol total & Experimentales & 74.00 & 4.58 & \multirow{2}{*}{3.99} & 0.002 \\
& Controles & 61,58 & 8.11 & & \\
Triglicéridos & Experimentales & 178.00 & 53.8 & 2.40 & 0.043 \\
& Controles & 107.00 & 60.0 & & \\
Col HDL & Experimentales & 34.58 & 4.16 & 2.15 & 0.075 \\
& Controles & 30.05 & 3.43 & & \\
Col LDL & Experimentales & 7.58 & 5.97 & -1.13 & 0.294 \\
\hline
\end{tabular}

La figura 2, podemos apreciar como cada rata comienza ligeramente con el aumento ponderal, llama la atención la rata número 3 , tiene un decline significativo de peso entre el día 16 y 19 con la recuperación de la ganancia ponderal ya al día 21.

En la figura 3, se observa el comportamiento de peso del GC señalándose en promedio un leve descenso promedio en la mayoría de todas las ratas $4.39 \%$ con respecto al peso inicial. Llama la aten-

53 ción la PDP promedio del $20 \%$ en los primeros 5 días para después recuperarlo.

En la tabla 3 se observa como la ES no provoca disminución significativa en el peso del GE a quien se le administró la solución de ES, en cuanto a los valores séricos de creatinina y colesterol HDL, no existió cambio significativo, sin embargo, un aumento significativo de los valores de urea, colesterol total, y triglicéridos del GE en relación al GC se presentó, tal y como se observa en la tabla. 


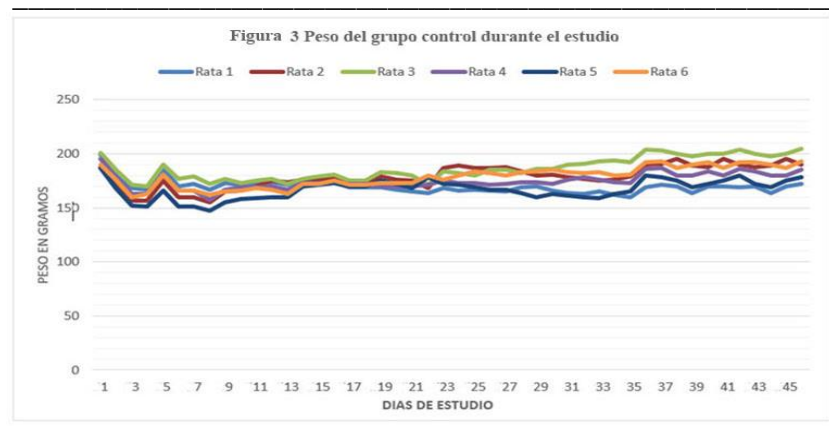

\section{Discusión}

La administración del alga ES en ratas señala que en la RP parece no ser eficaz tomándola como suplemento alimenticio, contrario a lo que reporto Becker et $\mathrm{al}^{23}$, en un estudio de doble ciego, con $2.8 \mathrm{~g}$ de ES suministrada tres veces al día durante un período de cuatro semanas presentaron una reducción pequeña pero estadísticamente significativa del peso corporal en pacientes ambulatorios obesos.

Cabe señalar que hay pocos estudios clínicos controlados sobre la PDP, que haya sido significativa, no obstante Villar et $\mathrm{al}^{1}$, Zarate et $\mathrm{al}^{8}$, atribuyen la PDP a un programa de alimentación y/o regímenes hipocalóricos utilizando ES como coadyuvante potencial para la supresión de apetito y antioxidante inmunodulador con buenos resultados. Mientras que para los vegetarianos, se propone como firme complemento de aminoácidos o fuentes proteicas ${ }^{14}$, en este estudio el mejoramiento significativamente de la urea respecto a los controles se sustenta la evidencia $^{14}$, respecto a que esta alga es una excelente fuente de aminoácidos al aumentar los niveles de urea, nuestros resultados también sugieren incluso su ingesta para la prevención de estos de déficit de niveles de colesterol y triglicéridos que pueden estar dados en situaciones especiales como desnutrición ${ }^{10}$.

Son varias referencias bibliográficas e instituciones como la, FAO, FDA y universidades que en el área de las ciencias biológicas han señalado efectos positivos inmunomoduladores Hirahashi et $\mathrm{al}^{6}$, antioxidantes Ruitang \& Te Jin ${ }^{16}$, efectos neuroendocrinológicos Sánchez et $\mathrm{al}^{7}$, y farmatoxicológicos Chamorro et $\mathrm{al}^{18}$, que aseguran sus propiedades como complemento alimentario sin mencionar su uso para la PDP como también consideramos para este estudio según los resultados de peso por lo que interpretamos los hallazgos como un suplemento que pudiera apoyar solo en el control de peso y mejorar estados nutricios endógenos con déficit. Al contrario de Chamorro et $\mathrm{al}^{11} \mathrm{y}$ Ruitang \& Te $\mathrm{Jin}^{16}$, en nuestro estudio hubo un ligero aumento de los niveles de triglicéridos, sin salir de los valores de referencia a diferencia de las ratas control quienes iniciaron incluso con niveles por debajo de estas y sus valores permanecieron estáticos o nunca mejoraron, una situación similar sucedió también con el colesterol total evaluado.

Al contrario de Ruitang \& Te $\mathrm{Jin}^{16}$ reporto a la ES como potente hipolipemiante, mejorando los niveles de $\mathrm{HDL}^{16}$, en este estudio el colesterol-LDL no mejoro y por el contrario, como ya se mencionó los niveles plasmáticos de los triglicéridos incrementaron $(\mathrm{p}=<0.043)$, cabe mencionar que las ratas tuvieron al inicio del estudio cerca del límite inferior del rango normal $(135 \mathrm{mg} / \mathrm{dL})$ y el resultado final no superó el rango del límite superior $200 \mathrm{mg} / \mathrm{dL}^{28}$. Otros estudios son necesarios ya que este estudio no es concluyente además que es importante diferenciar si existen cambios en la composición de la masa grasa y de la masa muscular de consumidores humanos en donde hay pocos estudios al respecto.

\section{Conflictos de intereses}

Se declara que no existe ningún conflicto de interés en la presente investigación. 
Agradecimientos

Damos las gracias a los asesores y al Departamento de Nutrición y especialmente al Departamento de Morfología al Dr. Francisco González Jaramillo por su colaboración y apoyo para hacer posible esta investigación.

\section{Aspectos éticos}

Se tuvo en cuenta las recomendaciones para el manejo de animales de experimentación según la Directiva Europea 2010/63/EU como referencia internacional, así como a nivel local la normativa de protección de animales de laboratorio la norma Oficial Mexicana NOM-062-ZOO-1999.

\section{Literatura citada}

1. de Villar NGP, Loria V, Monereo S. Tratamientos «alternativos» de la obesidad: mito y realidad. Med Clin (Barc) 2003;121(13):500-10. DOI: https://doi.org/10.1016/S0025-7753(03)74001-9

2. Karkos PD, Leong SC, Karkos CD, Sivaji N, Assimakopoulos DA. Spirulina in clinical practice: Evidence-based human applications. Evid Based Complement Alternat Med 2008;eCAM:1

3. Jiménez Escrig A, Goñi Cambrodón I. Evaluación nutricional y efectos fisiológicos de macroalgas marinas comestibles. Arch Latinoamer Nutr 1999;(49) 2:114-20.

4. Ramírez Moreno L, Olvera Ramírez R. Uso tradicional y actual de spirulina sp. (Arthrospira sp.). Interciencia 2006;31(9):657-63.

5. Benages IA, Pizzorno MT. Espirulina. Acta Farm Bonaer 1982;1(2):109-10.

6. Hirahashi T, Matsumoto M. Hazeki K, Saeki Y, Ui M, Seya T. Activation of the human innate 55 immune system by Spirulina: augmentation of interferon production and NK citotoxicity by oral administration of hot water extract of Spirulina platensis. Int Immunopharmacol 2002;2(4):42334. DOI: http://doi.org/10.1016/s1567-5769(01)0 $\underline{0166-7}$

7. Sánchez N, Bu M, León N, Pérez Saad H. Fundamentos de una posible acción beneficiosa de la Spirulina platensis en las neuropatías periféricas. Rev Cubana Plant Med 2002;7(3):146-50.

8. Zárate A, Saucedo R, Basurto L. Sustitutos no farmacéuticos que se usan para reducir el peso corporal. Acta Médica Grupo Ángeles 2005;3(2): 115-7.

9. Martínez Álvarez JR, Gómez Candela C, Villarino Marín A. Obesidad y alimentos funcionales: ¿son eficaces los nuevos ingredientes y productos? Rev Med Univ Navarra 2006;50(4):31-8.

10.Gutiérrez Salmeán G, Fabila Castillo L, Chamorro Cevallos G. Nutritional and toxicolo gical aspects of Spirulina (Arthrospira). Nutr Hosp 2015;32(1):34-40. DOI: http://doi.org/10. 3305/nh.2015.32.1.9001

11.Chamorro G, Salazar M, Gomes de Lima Araujo K, Pereira dos Santos C, Ceballos G, Fabila Castillo L. Actualización en la farmacología de Spirulina (Arthrospira), un alimento no convencional. Arch Latinoam Nutr 2002;52(3):232-40.

12.Wan D, Wu Q, Kuča K. Spirulina. In: Nutraceu ticals. Efficacy, Safety and Toxicity. Elsevier; 2016.p.569-83. DOI: https://doi.org/10.1016/ B978-0-12-802147-7.00042-5

13. Vo T-S, Ngo D-H, Kim S-K. Nutritional and pharmaceutical properties of microalgal Spirulina. In: Handbook of Marine Microalgae [Internet]. Elsevier; 2015. p. 299-308. DOI: https://doi.org/10.1016/B978-0-12-800776-1.000 $\underline{19-4}$ 
14.Kumar HD. Management of nutritional and health needs of malnourished and vegetarian people in India. In: Advances in experimental medicine and biology [Internet]. United States; 2004 [cited 2020 Jan 17]. p. 311-21. Available from: http://link.springer.com/ 10. 1007/978-1-4757-4820-8_23

15.Madhubalaji CK, Rashmi V, Chauhan VS, Dharmesh SM, Sarada R. Improvement of vitamin $\mathrm{B}_{12}$ status with Spirulina supplemen tation in Wistar rats validated through functional and circulatory markers. J Food Biochem. 2019;43(11):e3038. DOI: https:// doi.org/10.1111/jfbc. 13038

16.Ruitang D, Te Jin C. Hypolipidemic, antioxidant and antiinflammatory activities of microalgae Spirulina. Cardiovas Ther 2010;28(4):e33-5. DOI: http://doi.org/10.1111/j.1755-5922.2010.00 200.x

17.Martínez Álvarez JR, Gómez Candela C, Villarino Marín A. Obesidad y alimentos funcionales: ¿son eficaces los nuevos ingredientes y productos? Rev Med Univ Navarra 2006;50(4):31-8.

18. Chamorro G, Salazar M, Favila L, Bourges H. Farmacología y toxicología del alga Spirulina. Rev Invest Clin 1996;48(5):389-99.

19.Ramírez Moreno L, Olvera Ramírez R. Uso tradicional y actual de spirulina sp. (Arthrospira sp.). Interciencia. 2006;31(9):657-63.

20.Jensen GS, Ginsberg DI, Drapeau C. Blue-green algae as a inmuno-enhancer and biomodulador. Winter 2001;3(4):20-4.

21.Dinu M, Vlasceanu G, Dune A, Rotaru G. Researches concerning the growth of nutritive value of the bread products through the spirulin a adding. J Environ Prot Ecol. 2012;13(2):660-5.
22.Laura K. Alga Espirulina. Integral 2010;364:701.

23.Becker EW, Jakober B, Luft D, Schmulling RM. Clinical and biochemical evaluations of the alga Spirulina with regard to its application in the treatment of obesity. A double-blind cross-over study. Nutr Rep Int 1986;33:565-74.

24.Heo MG, Choung SY. Anti-obesity effects of Spirulina maxima in high fat diet induced obese rats via the activation of AMPK pathway and SIRT1. Food Funct. 2018;9(9):4906-15. DOI: http://doi.org/10.1039/c8fo00986d

25. Sotiroudis TG, Sotiroudis GT. Health aspects of Spirulina (Arthrospira) microalga food supple ment. J Serb Chem Soc 2013;78(3):395-405. DOI: http://doi.org/10.2298/JSC121020152S

26. Shao W, Ebaid R, El Sheekh M, Abomohra A, Eladel H. Pharmaceutical applications and consequent environmental impacts of Spirulina (Arthrospira): An overview. Grasas y Aceites 2019;70(1):e292. DOI: http://doi.org/10.3989/ gya.0690181

27.Norma Oficial Mexicana. NOM-062-ZOO-1999, Especificaciones técnicas para la producción, cuidado y uso de los animales de laboratorio [Internet]. Normas Mexicanas. DGN; 1999 [citado Jul 19 de 2019]. Disponible en: www.ibt.unam.mx/computo/pdfs/bioterio.NOM062.pdf

28. Sotiroudis TG, Sotiroudis GT. Health aspects of Spirulina (Arthrospira) microalga food supplement. J Serb Chem Soc 2013;78(3):395405. DOI: http://doi.org/10.2298/JS C12102015 $\underline{2 S}$

Nota del Editor:

Journal of the Selva Andina Research Society (JSARS) se mantiene neutral con respecto a los reclamos jurisdiccionales publicados mapas y afiliaciones institucionales. 